\title{
COMUNICAÇÃO BREVE Insatisfação, checagem corporal e comportamentos de risco para transtornos alimentares em estudantes de cursos da saúde
}

\author{
Body dissatisfaction, body-checking and risk behaviors for \\ eating disorders of undergraduate students of health area
}

Pedro Leopoldo de Oliveira', Maria Elisa Caputo Ferreira², Clara Mockdece Neves³, Juliana Fernandes Filgueiras Meireles ${ }^{3}$, Pedro Henrique Berbert de Carvalho ${ }^{4}$

\section{RESUMO}

Objetivos: Comparar os níveis de insatisfação corporal, checagem corporal e comportamentos de risco para transtornos alimentares entre graduandas de diferentes cursos da área de saúde, bem como avaliar a associação da checagem corporal e a insatisfação corporal, em cada curso de graduação. Métodos: Participaram 92 universitárias com idade entre 18 e 35 anos, da cidade de Governador Valadares, Minas Gerais, que responderam a instrumentos de autorrelato sobre: dados sociodemográficos, insatisfação corporal (Body Shape Questionnaire), comportamentos de checagem corporal (Body Checking Questionnaire) e comportamentos de risco para transtornos alimentares (Eating Attitudes Test-26). Foi realizada estatística descritiva, inferencial (Kruskal-Wallis) e teste de correlação de Spearman utilizando o software SPSS 21.0 e adotando nível de significância de 5\%. Resultados: Os testes de comparação não demonstraram diferenças para a insatisfação corporal $(H(4,92)=3,534260, p=0,47)$, checagem corporal $(H(4,92)=3,154817, p=0,53)$ e comportamentos de risco para transtornos alimentares $(H(4,92)=4,435904, p=0,35)$ entre os cursos analisados. Foi encontrada associação significativa das variáveis checagem corporal e insatisfação corporal para quase todos os cursos, exceto Nutrição $\left(r_{\text {spearman }}=0,01, p>0,05\right)$, sendo Farmácia aquele que apresentou relação forte entre essas variáveis $\left(r_{\text {spearman }}\right.$ superior a 0,80$)$. Conclusões: Conclui-se não haver diferenças significativas para as variáveis insatisfação corporal, checagem corporal e comportamentos de risco para transtornos alimentares entre as estudantes dos cursos da área de saúde. Destaca-se, contudo, variações na importância de algumas variáveis, como a checagem corporal, na construção da imagem corporal dos sujeitos.

\section{ABSTRACT}

Objectives: To compare body dissatisfaction, body-checking behaviors and risk behaviors for eating disorders among undergraduate students of different courses of health area, and to evaluate the relationship of body-checking behaviors and body dissatisfaction in each course. Methods: A total of 92 undergraduate students aged between 18 and 35 years old

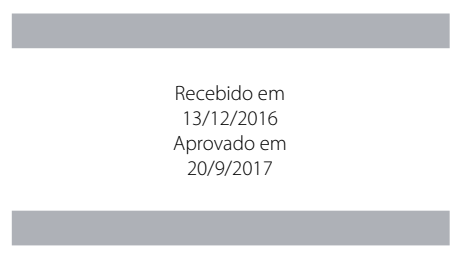

DOI: $10.1590 / 0047-2085000000174$
1 Universidade Federal de Juiz de Fora (UFJF), Campus Governador Valadares (GV), Curso de Bacharelado em Educação Física. 2 Universidade Federal de Juiz de Fora (UFJF), Faculdade de Educação Física e Desportos, Programa de Pós-Graduação em Educação Física. 3 Universidade Federal de Juiz de Fora (UFJF), Instituto de Ciências Humanas, Programa de Pós-Graduação em Psicologia.

4 Universidade Federal de Juiz de Fora (UFJF), Campus Governador Valadares (GV), Instituto de Ciências da Vida, Departamento de Educação Física.

Endereço para correspondência: Pedro Henrique Berbert de Carvalho Rua Manoel Byrro, 241, Vila Bretas 35032-620 - Governador Valadares, MG, Brasil

E-mail: pedro.berbert@uff.edu.br 


\section{Keywords}

Body image, feeding and eating disorders, risk groups, students. from the city of Governador Valadares, Minas Gerais, Brazil, took part in this study. They filled self-report instrument about sociodemographic data, body dissatisfaction (Body Shape Questionnaire), body-checking behaviors (Body Checking Questionnaire) and risk behaviors for eating disorders (Eating Attitudes Test-26). Descriptive, inferential (Kruskal-Wallis test) and correlational (Spearman's Rank test) statistics were performed using SPSS 21.0 software and adopting a significance level of 5\%. Results: Comparison tests did not show differences in body dissatisfaction $(H(4,92)=3.534260, p=0.47)$, body-checking behavior $(H(4,92)=$ $3.154817, p=0.53)$ or risk behaviors for eating disorders $(H(4,92)=4.435904, p=0.35)$ among the courses analyzed. There was a significant association between body-checking and body dissatisfaction for almost all courses, except for Nutrition $\left(r_{\text {spearman }}=0.01, p>0.05\right)$, with Pharmacy having a strong relationship between those variables $\left(r_{\text {spearman }}\right.$ higher than 0.80$)$. Conclusions: It was concluded that there are no significant differences in body dissatisfaction, body-checking or risk behaviors for eating disorders among health area courses. We emphasize that some variables demonstrate differentiated importance in the construction of the subjects' body image, such as body-checking behaviors.

\section{INTRODUÇÃO}

A imagem corporal pode ser entendida como a imagem mental, os sentimentos, os pensamentos e os comportamentos concernentes ao corpo como um todo, ou mesmo às partes que o constituem ${ }^{1,2}$, destacando-se o número de pesquisas relacionadas à insatisfação corporal, sentimento negativo que o indivíduo tem em relação ao seu peso e à sua forma corporal ${ }^{1,2}$.

Diversos fatores prejudiciais à saúde estão relacionados à insatisfação corporal, como o uso de substâncias anorexígenas, esteroides anabólicos, abuso de laxantes, entre outros ${ }^{3}$. Tais comportamentos de risco justificam a atenção de profissionais de saúde com relação à insatisfação corporal ${ }^{4}$.

Indivíduos insatisfeitos com o próprio corpo estão propensos a restrição alimentar e comportamentos de checagem corporal contínuos ${ }^{3}$. A checagem corporal é definida como um comportamento repetitivo de autoavaliação corporal ${ }^{3}$. Vale destacar que algumas variáveis podem impactar a imagem corporal de jovens universitários, como, por exemplo, o curso de graduação em que o sujeito está matriculado $^{5}$. Existe uma crença de que estudantes dos cursos de Educação Física e Nutrição sejam aqueles que apresentam níveis mais elevados de insatisfação corporal e maior risco para o desenvolvimento de transtornos alimentares ${ }^{5}$.

Todavia, os estudos que realizam tais afirmações ${ }^{5-10}$ não se valeram de uma comparação direta dos níveis de insatisfação corporal ou comportamentos de risco para transtornos alimentares entre grupos de diferentes cursos de graduação, o que impossibilita afirmar que os cursos de Educação Física e Nutrição são grupos de risco.

O objetivo principal do presente estudo é a comparação da insatisfação corporal, checagem corporal e comportamentos de risco para transtornos alimentares entre diferen- tes cursos de graduação da área de saúde. Como objetivo secundário, busca-se avaliar a associação das variáveis checagem corporal e insatisfação corporal, em cada curso de graduação, dado ainda insipiente na literatura científica.

\section{MÉTODOS}

\section{Amostra}

Este estudo do tipo transversal foi desenvolvido com uma amostra por conveniência de 129 estudantes do sexo feminino de Instituições de Ensino Superior da cidade de Governador Valadares, Minas Gerais. O critério de inclusão era estar regularmente matriculada em qualquer período de um curso de graduação, ser maior de 18 anos, estar presente no momento da coleta e aceitar participar voluntariamente da pesquisa. Como critérios de exclusão foram utilizados: idade superior a 35 anos e deixar parcial ou totalmente um dos questionários sem responder.

\section{Aspectos éticos}

O projeto de pesquisa foi aprovado pelo Comitê de Ética e Pesquisa em Seres Humanos da Universidade Federal de Juiz de Fora, com o número de inscrição 26738014.0.0000.5147 e parecer de aprovação número 1.561.592. Todos os participantes concordaram em participar da pesquisa e assinaram o Termo de Consentimento Livre e Esclarecido (TCLE).

\section{Instrumentos}

\section{Caracterização da amostra}

Para caracterizar a amostra, foi utilizado um questionário sociodemográfico que investiga as informações sobre idade e curso de graduação em que se encontra matriculada. 


\section{Insatisfação com a imagem corporal}

A insatisfação e as preocupações com a forma do corpo foram avaliadas pela aplicação do Body Shape Questionnaire (BSQ) ${ }^{11}$. Adotou-se a versão curta do BSQ, validada para jovens brasileiras, composta por oito itens ${ }^{11}$ (itens $5,11,15,20$, 21, 22, 25 e 28), respondidos em escala na forma Likert de 6 pontos (1 - nunca a 6 - sempre), variando de 6 a 36 pontos ${ }^{11}$. Quanto maior a pontuação, maior é a insatisfação corporal do indivíduo. A consistência interna do BSQ obtida nesta pesquisa foi adequada $(a=0,92)$.

\section{Comportamento de checagem corporal}

A frequência do comportamento de checagem corporal foi avaliada pela versão validada para jovens adultas brasileiras do Body Checking Questionnaire (BCQ)'12. O BCQ é composto por 12 itens, respondidos em escala do tipo Likert de pontos ( 1 - nunca a 5 - muito frequentemente). $O$ resultado pode variar de 12 a 60 pontos. Quanto maior a pontuação, mais frequente é a checagem corporal. Na presente pesquisa foi verificado a igual a 0,87 .

\section{Comportamentos de risco para transtornos alimentares}

Os comportamentos de risco para transtornos alimentares foram avaliados pela aplicação da versão brasileira do Eating Attitudes Test (EAT-26) ${ }^{13}$. O EAT-26 é composto por 26 questões de autorrelato, respondidas em uma escala Likert de pontos que varia de sempre (3 pontos) a nunca (0 ponto), exceto a questão 25, que apresenta pontuação inversa. Escore superior ou igual a 21 pontos salienta possíveis comportamentos de risco para o transtorno alimentar (EAT-26 positivo) ${ }^{13}$. A consistência interna do EAT-26 nesta pesquisa foi igual a 0,82 .

\section{Procedimentos}

Foi realizado contato com diversos cursos das ciências da saúde da cidade de Governador Valadares, e ao final agendadas coletas com cinco cursos: Medicina, Educação Física, Nutrição, Fisioterapia e Farmácia. Foi estabelecido contato com os professores e explicados os procedimentos da pesquisa. Para as participantes, foram explicados os objetivos e procedimentos da pesquisa e entregue o protocolo de pesquisa formado pelos quatro instrumentos de avaliação supracitados. As participantes responderam aos questionários, sem limite de tempo definido. Nenhuma informação adicional foi fornecida.

\section{Análise estatística}

Foi realizada análise descritiva (média, desvio-padrão, valores mínimo e máximo). Os dados não apresentaram distribuição normal (verificado pelo teste Kolmogorov-Smirnov). A diferença entre as variáveis contínuas foi verificada por meio do teste Kruskal-Wallis. Spearman-Rank teste foi utilizado para análise de associações entre as variáveis checagem e insatisfação corporal. A consistência interna dos instrumentos foi avaliada pelo alfa de Cronbach, e considerado adequado se superior a 0,70. O nível de significância adotado foi de 5\% $(p<0,05)$ e o software utilizado o SPSS 21.0.

\section{RESULTADOS}

Esta pesquisa contou com a participação de 129 universitárias, e, após exclusão de alguns casos com base nos critérios adotados, foram analisados os dados de 92 estudantes. A Tabela 1 apresenta os valores das análises descritivas e comparativas das variáveis estudadas.

A partir do teste de comparações entre os grupos para as variáveis analisadas, foram identificadas diferenças estatísticas apenas para a idade $(H(4,92)=13,02828, p=0,01)$ entre Educação Física e Medicina, sendo este último grupo o mais jovem. Os testes de comparação para as variáveis insatisfação corporal $(H(4,92)=3,534260, p=0,47)$, checagem corporal $(H(4,92)=3,154817, p=0,53)$ e comportamentos de risco para transtornos alimentares $(H(4,92)=4,435904$, $p=0,35)$ não demonstraram diferenças entre os grupos analisados.

A Tabela 2 apresenta os valores encontrados a partir do teste de associação de Spearman-Rank entre a insatisfação e a checagem corporal dividida por cursos.

\section{DISCUSSÃO}

De acordo com os achados na literatura, foram identificados diversos estudos sobre insatisfação corporal em estudantes universitários ${ }^{3-11}$. Alguns desses apontam as estudantes dos cursos de Educação Física e Nutrição como grupos de risco para distúrbios de imagem corporal e transtornos alimentares ${ }^{6-10}$. Miranda et al. ${ }^{14}$ e Bosi et al. ${ }^{15}$ supõem que alunos de alguns cursos de graduação podem estar mais sujeitos à busca do padrão de corpo exposto pela mídia, pois convivem diretamente com a melhora da aparência física, o que seria o caso dos estudantes de Educação Física e Nutrição.

Porém, esses estudos não se valeram de comparação direta entre os escores obtidos pelos estudantes de Educação Física e Nutrição com demais cursos. Logo, o presente estudo teve como objetivo principal a comparação entre os cursos de graduação, levando em conta as variáveis insatisfação corporal, checagem corporal e comportamentos de risco para transtornos alimentares.

Não foi encontrada diferença significativa entre os grupos analisados para a variável insatisfação corporal, resultado este que diverge de estudos anteriores ${ }^{5-10}$. Conforme aponta Souza e Alvarenga ${ }^{5}$, os cursos de Educação Física e Nutrição 
Tabela 1. Análise descritiva e comparativa das variáveis idade (anos), insatisfação corporal, checagem corporal e comportamentos de risco para transtornos alimentares divididos entre os cursos da área de saúde

\begin{tabular}{|c|c|c|c|c|c|c|}
\hline Variável estatística & Medicina $(n=29)$ & Educação Física ( $\mathrm{n}=23$ ) & Nutrição $(n=15)$ & Fisioterapia $(n=17)$ & Farmácia $(n=8)$ & p-valor \\
\hline \multicolumn{7}{|l|}{ Idade } \\
\hline Média \pm DP & $19,58 \pm 2,14^{\mathrm{a}}$ & $24,60 \pm 5,15^{a}$ & $22,60 \pm 6,06$ & $21,00 \pm 2,64$ & $20,75 \pm 3,01$ & $0,01^{*}$ \\
\hline Mín-Máx & $18-29$ & $18-34$ & $18-35$ & $19-28$ & $18-27$ & \\
\hline \multicolumn{7}{|l|}{ BSQ } \\
\hline Média \pm DP & $21,75 \pm 8,26$ & $24,13 \pm 10,08$ & $23,53 \pm 9,78$ & $28,29 \pm 12,24$ & $23,62 \pm 9,98$ & 0,47 \\
\hline Mín-Máx & $9-44$ & 11-48 & 8-40 & 10-47 & 8-38 & \\
\hline \multicolumn{7}{|l|}{$\mathrm{BCQ}$} \\
\hline Média \pm DP & $15,93 \pm 8,03$ & $19,04 \pm 9,57$ & $21,86 \pm 10,92$ & $20,52 \pm 12,60$ & $15,62 \pm 8,15$ & 0,35 \\
\hline Mín-Máx & $15-40$ & $16-47$ & $16-42$ & 13-48 & 13-31 & \\
\hline \multicolumn{7}{|l|}{ EAT } \\
\hline Média \pm DP & $6,86 \pm 2,69$ & $6,17 \pm 2,38$ & $5,46 \pm 2,06$ & $7,00 \pm 3,79$ & $6,62 \pm 2,55$ & 0,53 \\
\hline Mín-Máx & 3-15 & 3-12 & 3-9 & 2-15 & $3-11$ & \\
\hline
\end{tabular}

n: número de estudantes; DP: desvio-padrão; Mín: valor mínimo; Máx: valor máximo; BSQ: Body Shape Questionnaire; BCQ: Body Checking Questionnaire; EAT-26: Eating Attitudes Test - 26.

* significativo para $p<0,05$.

a Diferença entre o grupo de estudantes de Medicina e Educação Física.

Tabela 2. Teste de associação de Spearman-Rank entre a insatisfação corporal e a checagem corporal, dividido por curso da área de saúde

\begin{tabular}{lccccc}
\hline Variáveis & Medicina $(\mathrm{n}=29)$ & Educação Física $(\mathrm{n}=23)$ & Nutrição $(\mathrm{n}=15)$ & Fisioterapia $(\mathrm{n}=17)$ & Farmácia $(\mathrm{n}=8)$ \\
\hline $\mathrm{BSQ} \times \mathrm{BCQ}$ & $0,52^{*}$ & $0,78^{*}$ & 0,01 & $0,58^{*}$ & $0,81^{*}$ \\
\hline
\end{tabular}

n: número de estudantes; $B S Q: B o d y$ Shape Questionnaire; $B C Q=B o d y$ Checking Questionnaire.

* significativo para $p<0,05$.

são os mais investigados no cenário nacional. A carência de investigações com demais cursos de graduação impossibilita uma comparação entre as frequências de indivíduos satisfeitos e insatisfeitos, ainda que tal comparação se dê de maneira indireta, ou seja, apenas pela observação dos percentuais ou escores de insatisfação.

Já para a variável checagem corporal, não foram encontradas diferenças significativas entre os grupos analisados, resultado de difícil comparação com demais estudos da literatura, uma vez que existe uma carência na área científica sobre a checagem corporal ${ }^{3}$. Carvalho et al. ${ }^{3}$, avaliando estudantes de diversos cursos de graduação, verificaram diferença entre os sexos para a checagem. As mulheres demonstraram maior frequência de checagem corporal. Contudo, não foi realizada comparação direta da checagem corporal entre os cursos de graduação ${ }^{3}$, o que dificulta a análise dos resultados apresentados pela presente pesquisa.

Acredita-se que esta seja a primeira investigação nacional a realizar análise de comparação dessa variável em estudantes universitárias brasileiras. É preciso ampliar o número de pesquisa sobre a checagem corporal neste público, considerando a associação desse construto com distúrbios de imagem corporal e transtornos alimentares ${ }^{3}$.

Quanto aos comportamentos de risco para transtornos alimentares, não foi encontrada diferença significativa entre os grupos analisados, resultado que diverge dos achados até então disponíveis ${ }^{5,6,9}$. Não é possível afirmar que a frequência observada nos comportamentos de risco para transtornos alimentares nos acadêmicos de Nutrição ou Educação Física seja maior ou menor do que a observada em outros cursos, senão pela comparação direta das frequências observadas.

A hipótese de que esses dois cursos possuem uma relação mais forte com conceitos como estética e aparência física pode não ser uma realidade. Por exemplo, estudantes de Fisioterapia estão imersos no contexto de sua profissão, que inclui atividades como Fisioterapia Dermatológica, Fisioterapia Estética, entre outros. Estudantes de Medicina estão em contato com procedimentos estéticos (cirurgia estética, por exemplo), bem como com o estudo de Psicopatologias (transtornos alimentares, por exemplo). Já estudantes de Farmácia possuem relação estreita com fármacos, dos quais podemos citar as substâncias anorexígenas. Assim, todos esses futuros profissionais, durante a graduação, estão em contato com conceitos, fundamentos e discussões sobre aspectos relacionados ao cuidado com o corpo. Esses podem ser os fatores que justificam os resultados da presente pesquisa quanto a não diferença entre os grupos para as variáveis investigadas.

Há ainda que se considerar que todos esses sujeitos estão expostos a um universo de informações globalizadas sobre saúde e estética². Sabe-se que a imagem corporal e os transtornos alimentares são influenciados por fatores socioculturais ${ }^{1}$, o que parece atingir de maneira similar todos os sujeitos.

Já com relação ao objetivo secundário desta pesquisa, foi encontrada relação significativa da checagem corporal e insatisfação corporal para quase todos os cursos, exceto Nutrição. Esse resultado não significa que os acadêmicos de 
Nutrição estão livres de insatisfação, mas sim que para este curso de graduação outros fatores intervenientes estão implicados na construção de uma imagem corporal negativa. Enquanto para os demais cursos o comportamento de checagem corporal contribuiu diretamente para a insatisfação, para a Nutrição, outros fatores não avaliados na presente investigação podem estar associados.

O construto imagem corporal é multifacetado ${ }^{1,2}$, o que significa que muitos outros fatores podem estar relacionados à insatisfação corporal. Cumpre, portanto, destacar a necessidade de investigação de possíveis fatores que expliquem a insatisfação corporal do curso de Nutrição, bem como ampliar as pesquisas sobre outros cursos da área de saúde, como Odontologia, Enfermagem, Biomedicina e demais.

Este estudo apresenta como limitação seu delineamento transversal, que não permite observar a relação de causa-efeito. Outra limitação se refere à amostra reduzida e centrada em estudantes de instituições de ensino superior de uma única cidade brasileira, não permitindo fazer inferência a todos os universitários brasileiros. Todavia, avanços foram obtidos por meio da comparação direta entre os diversos cursos, ampliando a compreensão sobre as diferenças deles com relação à insatisfação corporal, checagem corporal e comportamentos de risco para transtornos alimentares. Ainda podemos citar como limitação a não inclusão de jovens do sexo masculino. Entretanto, há de se considerar as idiossincrasias dessa população e a necessidade de utilização de instrumentos específicos de avaliação da imagem corporal e transtornos alimentares nesse público. Estudos deste tipo também são importantes, pois esses são os futuros profissionais que irão lidar com o público, sendo que suas preocupações com o próprio corpo podem refletir na sua prática profissional e influenciar seus alunos ou clientes.

\section{CONCLUSÃO}

Os resultados apresentados neste estudo demonstram não haver diferenças significativas para a insatisfação corporal, checagem corporal e os comportamentos de risco para transtornos alimentares entre estudantes dos cursos de Medicina, Educação Física, Nutrição, Fisioterapia e Farmácia. Em adição, foi observada moderada e alta associação entre checagem e insatisfação corporal para os cursos avaliados, exceto Nutrição.

\section{CONTRIBUIÇÕES INDIVIDUAIS}

\footnotetext{
Pedro Leopoldo de Oliveira e Pedro Henrique Berbert de Carvalho - Foram responsáveis pela concepção e desenho do estudo, análise e interpretação dos dados, elaboração, revisão e aprovação da versão final.
}

Clara Mockdece Neves, Juliana Fernandes Filgueiras Meireles e Maria Elisa Caputo Ferreira - Contribuíram substancialmente na elaboração do artigo, revisão crítica de seu conteúdo e aprovação da versão final.

\section{CONFLITOS DE INTERESSE}

Os autores não possuem conflitos de interesses a serem declarados.

\section{AGRADECIMENTOS}

Aos estudantes das Instituições de Ensino Superior participantes e a Universidade Federal de Juiz de Fora, pela bolsa de iniciação científica (BIC) ofertada ao primeiro autor deste trabalho.

\section{REFERÊNCIAS}

1. Cash TF, Smolak L. (Ed.). Body image: a handbook of science, practice, and prevention. London: The Guilford Press; 2011.

2. Ferreira MEC, Castro MR, Morgado FFR. Imagem corporal: reflexões, diretrizes e práticas de pesquisa. Juiz de Fora (MG): Editora UFJF; 2014.

3. Carvalho PHB, Filgueiras JF, Neves CM, Coelho FD, Ferreira ME. Checagem corporal, atitude alimentar inadequada e insatisfação com a imagem corporal de jovens universitários. J Bras Psiquiatr. 2013;2(2):108-14.

4. Silva TR, Saenger G, Pereira EF. Fatores associados à imagem corporal em estudantes de Educação Física. Motriz Rev Educ Fís. 2011;17(4):630-9.

5. Souza AC, Alvarenga MS. Insatisfação com a imagem corporal em estudantes universitários: uma revisão integrativa. J Bras Psiquiatr. 2016;65(3):286-99.

6. Bosi MLM, Luis RR, Morgado CMC, Costa MLS, Carvalho RJ. Autopercepção da imagem corporal entre estudantes de nutrição: um estudo no município do Rio de Janeiro. J Bras Psiquiatr. 2006:55(2):108-13.

7. Ferrari EP, Petroski EL, Silva DA. Associação entre percepção da imagem corporal e estágios de mudança de comportamento em acadêmicos de educação física. Rev Bras Cineantropom Desempenho Hum. 2012;14(5):535-44.

8. Quioca T, Lange F, Grigollo LR, Noradi Junior RJ. Percepção da imagem e saúde corporal dos universitários do Curso de Educação Física da Unoesc de Joaçaba, SC. Evid. (Joaçaba, Online). 2010;10(1):43-56.

9. Silva JD, Silva AB, Oliveira AV, Nemer AS. Influência do estado nutricional no risco para transtornos alimentares em estudantes de nutrição. Ciên Saúde Colet. 2012;17(12):3399-406.

10. Ferrari EP, Petroski EL, Silva DA. Prevalence of body image dissatisfaction and associated factors among physical education students. Trends Psychiatry Psychother. 2013;35(2):119-27.

11. Silva WR, Dias JCR, Maroco J, Campos JADB. Confirmatory factor analysis of different versions of the Body Shape Questionnaire applied to Brazilian university students. Body Image. 2014;11(4):384-90

12. Campana ANNB. Tradução, adaptação transcultural e validação do "Body Image Avoidance Questionnaire (BIAQ)" e do "Body Checking Questionnaire (BCQ)" para a língua portuguesa no Brasil [mestrado]. Campinas: Universidade Estadual de Campinas; 2007.

13. Nunes MA, Camey S, Olinto MTA, Mari JJ. The validity and 4-year test-retest reliability of the Brazilian version of the Eating Attitudes Test-26. Braz J Med Biol Res. 2005;38(11):1655-62.

14. Miranda VPN, Filgueiras JF, Neves CM, Teixeira PC, Ferreira MEC. Insatisfação corporal em universitários de diferentes áreas de conhecimento. J Bras Psiquiatr. 2012;61(1):25-32.

15. Bosi MLM, Luiz RR, Uchimura KY, Oliveira FP. Comportamento alimentar e imagem corporal entre estudantes de educação física. J Bras Psiquiatr. 2008;57(1):28-33. 